\title{
A Texture Based Model for Polycrystal Plasticity
}

\author{
Thomas Böhlke'1, Gerrit Risy and Albrecht Bertram \\ Institut für Mechanik, Otto-von-Guericke-Universität Magdeburg \\ Postfach 4120, D-39016 Magdeburg, Germany \\ ${ }^{1}$ Ph. 0049-391-67-12592 Fax 0049-391-67-12863 Email thomas.boehlke@mb.uni-magdeburg.de
}

Keywords: anisotropic viscoplasticity, crystallite orientation distribution function, earing behavior, finite element method, texture components, texture induced plastic anisotropy

\begin{abstract}
In the present work the texture component model developed in [2] is used to predict the plastic anisotropy of a textured aluminum sheet in terms of the yield stress, the $R$ value, and the earing profile resulting from a cup drawing operation. The model which is formulated in a viscoplastic setting, has been implemented in the commercial finite element code ABAQUS. The stress deviator is computed directly from the crystallite orientation distribution function which is approximated by a set of Gauss type model functions. The model is particularly suitable for the description of crystallographic textures which can be described by a small number of texture components. The numerical results are compared with experimental data and numerical results by Lege et al. [8].
\end{abstract}

\section{Introduction}

An inhomogeneous distribution of crystal orientations induces anisotropies of the macroscopic mechanical behavior. Therefore an accurate description of this distribution plays a significant role if the anisotropy of sheet metals has to be taken into account in finite element simulations. The relation between the macroscopic stress and strain measures and the crystallographic texture can be determined, e.g., based on Taylor type models or self-consistent schemes. The former group of models is widely used [7] because it is computationally less expensive. Taylor type models give a reasonable qualitative approximation of the crystallographic texture evolution in single-phase face-centered cubic materials with high stacking-fault energy such as copper and aluminum. One of the most significant shortcoming of this model class is the overestimation of the sharpness of the texture and hence of the mechanical anisotropy. This overestimation is very pronounced if the codf is discretized by a small set of discrete crystal orientations. In the present paper we consider a Taylor type model based on texture components, each of which is approximated by a central distribution. The effective stress tensor is determined by an integration of the single crystal stress state over the orientation space. It is shown that the texture component model allows for an accurate description of the yield stress, the $R$ value, and the earing profile even if only a small number of crystal orientations is taken into account.

\section{Homogenization of the Stress Tensor}

For the homogenization of the Kirchhoff stress tensor $\tau$ it is assumed that the polycrystal deforms homogeneously, i.e. $\bar{J}=J, \bar{D}=\boldsymbol{D}$, where $J$ and $\boldsymbol{D}^{\prime}$ denote the determinant of the 
deformation gradient and the strain rate deviator, respectively. In the following we adopt the assumption that dilatations are purely elastic, whereas distortions are purely viscoplastic. Both deformation modes are assumed to be decoupled. A detailed description of the model can be found in [2]. The spherical part and the deviatoric part of the stress tensor $\tau$ are denoted by $\tau^{\circ}$ and $\tau^{\prime}$, respectively. For the volume averages over the initial placement we have

$$
\bar{\tau}=\frac{1}{V} \int_{V}\left(\tau^{\circ}+\tau^{\prime}\right) \mathrm{d} V=\bar{\tau}^{\circ}+\bar{\tau}^{\prime}
$$

The spherical part $\overline{\boldsymbol{\tau}}^{\circ}$ is associated with volume changes and is modeled by the following finite elastic law [10]

$$
\overline{\boldsymbol{\tau}}^{\circ}=\frac{K}{2}\left(\bar{J}^{2}-1\right) \boldsymbol{I}
$$

where $K$ denotes the bulk modulus. The deviatoric part of the stress tensor $\bar{\tau}^{\prime}$ is associated with shape changes and is assumed to depend on the distribution of the crystal orientations $\boldsymbol{Q} \in S O(3)$ and on the distribution of the critical resolved shear stresses $\tau^{C} \in \mathbb{R}^{+}$. The state of the polycrystal is described by a distribution function $h\left(\boldsymbol{Q}, \tau^{C}\right)$

$$
\frac{\mathrm{d} V}{V}\left(\boldsymbol{Q}, \tau^{C}\right)=h\left(\boldsymbol{Q}, \tau^{C}\right) \mathrm{d} Q \mathrm{~d} \tau^{C}
$$

which specifies the volume fraction of crystals having the orientation $\boldsymbol{Q}$ and the critical resolved yield stress $\tau^{C}$. The distribution function $h\left(\boldsymbol{Q}, \tau^{C}\right)$ is non-negative and normalized in the sense of

$$
h\left(\boldsymbol{Q}, \tau^{C}\right) \geq 0 \quad \forall \boldsymbol{Q} \in S O(3), \tau^{C} \in \mathbb{R}^{+}, \quad \int_{\mathcal{H}} h\left(\boldsymbol{Q}, \tau^{C}\right) \mathrm{d} Q \mathrm{~d} \tau^{C}=1,
$$

where $\mathcal{H}=\mathbb{R}^{+} \times S O(3)$. The stress tensor $\overline{\boldsymbol{\tau}}^{\prime}$ is computed by means of an integration over $\mathcal{H}$

$$
\overline{\boldsymbol{\tau}}^{\prime}=\int_{\mathcal{H}} h\left(\boldsymbol{Q}, \tau^{C}\right) \boldsymbol{\tau}^{\prime}\left(\overline{\boldsymbol{D}}^{\prime}, \boldsymbol{Q}, \tau^{C}\right) \mathrm{d} Q \mathrm{~d} \tau^{C}
$$

The stress state $\tau^{\prime}$ and the lattice spin $\dot{Q} Q^{-1}$ can be computed by the following equations

$$
\begin{aligned}
& \mathbf{0}=\boldsymbol{D}^{\prime}-\boldsymbol{Q} \operatorname{sym}\left(\tilde{\boldsymbol{K}}\left(\boldsymbol{Q}^{\top} \boldsymbol{\tau}^{\prime} \boldsymbol{Q}, \tau^{C}\right)\right) \boldsymbol{Q}^{\top}, \\
& \dot{\boldsymbol{Q}} \boldsymbol{Q}^{-1}=\boldsymbol{W}-\boldsymbol{Q} \operatorname{skw}\left(\tilde{\boldsymbol{K}}\left(\boldsymbol{Q}^{\top} \boldsymbol{\tau}^{\prime} \boldsymbol{Q}, \tau^{C}\right)\right) \boldsymbol{Q}^{\boldsymbol{\top}}
\end{aligned}
$$

[5], where the function $\tilde{\boldsymbol{K}}$ is defined by

$$
\tilde{\boldsymbol{K}}\left(\boldsymbol{Q}^{\top} \boldsymbol{\tau}^{\prime} \boldsymbol{Q}, \tau^{C}\right)=\sum_{\alpha=1}^{N} \dot{\gamma}_{\alpha}\left(\tau_{\alpha}, \tau^{C}\right) \tilde{\boldsymbol{M}}_{\alpha}, \quad \dot{\gamma}_{\alpha}\left(\tau_{\alpha}, \tau^{C}\right)=\dot{\gamma}_{0} \operatorname{sign}\left(\tau_{\alpha}\right)\left|\frac{\tau_{\alpha}}{\tau^{C}}\right|^{m} .
$$

$\tau_{\alpha}=\left(\boldsymbol{Q}^{\top} \boldsymbol{\tau}^{\prime} \boldsymbol{Q}\right) \cdot \tilde{\boldsymbol{M}}_{\alpha}$ denotes the resolved shear stress in the $\alpha$-th slip system. $\tilde{\boldsymbol{M}}_{\alpha}$ is the Schmid tensor. The evolution of the hardening variable $\tau^{C}$ is specified by the Kocks-Mecking model $[6,2]$. 


\section{Approximation of the CODF by Texture Components}

There are many crystallographic textures which can be described by a small number of texture components or fibers $[11,3,4,7]$. In this work only texture components are considered. A texture component is a crystal orientation for which the codf shows a (local) maximum in the elementary region. In the neighborhood, the codf is decreasing in an isotropic or anisotropic way. In the following the distribution function $h\left(\boldsymbol{Q}, \tau^{C}\right)$ is decomposed into an isotropic part $h^{I}$ and an anisotropic part $h^{A}$ with volume fractions $\nu^{I}$ and $\nu^{A}$. The anisotropic part is modeled as a superposition of $N_{c}$ central distributions $g\left(\boldsymbol{Q}, \boldsymbol{Q}_{\alpha}, b_{\alpha}\right)$ with mean orientations $\boldsymbol{Q}_{\alpha}$ and volume fractions $\nu_{\alpha}^{A}\left(\alpha=1, \ldots, N_{c}\right)$. The hardening state of each of the components is assumed to be homogeneous and hence can be specified in terms of one drag stress $\tau_{\alpha}^{C}$. The isotropic part depends on the arithmetic mean of the drag stresses $\bar{\tau}^{C}=\sum_{\alpha=1}^{N_{c}} \nu_{\alpha}^{A} \tau_{\alpha}^{C}$. As a result, we have

$$
h\left(\boldsymbol{Q}, \tau^{C}\right)=\nu^{I} h^{I}\left(\bar{\tau}^{C}\right)+\nu^{A} h^{A}\left(\boldsymbol{Q}, \boldsymbol{Q}_{\alpha}, b_{\alpha}, \tau_{\alpha}^{C}\right),
$$

where

$$
h^{I}\left(\bar{\tau}^{C}\right) \sim \delta_{\bar{\tau}^{C}}, \quad h^{A}\left(\boldsymbol{Q}, \boldsymbol{Q}_{\alpha}, b_{\alpha}, \tau_{\alpha}^{C}\right) \sim \sum_{\alpha=1}^{N_{c}} \nu_{\alpha}^{A} g\left(\boldsymbol{Q}, \boldsymbol{Q}_{\alpha}, b_{\alpha}\right) \delta_{\tau_{\alpha}^{C}} .
$$

The constraints upon the volume fractions are

$$
\nu^{I}+\nu^{A}=1, \quad \sum_{\alpha=1}^{N_{c}} \nu_{\alpha}^{A}=1 .
$$

The value of a central distribution $g\left(\boldsymbol{Q}, \boldsymbol{Q}_{\alpha}, b_{\alpha}\right)$ at $\boldsymbol{Q}$ only depends on the distance $\omega$ between $\boldsymbol{Q}$ and $\boldsymbol{Q}_{\alpha}$. The distance between two crystal orientations is generally defined as

$$
\omega\left(\boldsymbol{Q}, \boldsymbol{Q}_{\alpha}\right)=\arccos \left(\frac{1}{2}\left(\operatorname{tr}\left(\boldsymbol{Q} \boldsymbol{Q}_{\alpha}^{-1}\right)-1\right)\right)
$$

[3]. A specific central distribution is the Mises-Fischer distribution

$$
g\left(\boldsymbol{Q}, \boldsymbol{Q}_{\alpha}, b_{\alpha}\right)=N\left(S_{\alpha}\right) e^{\left(S_{\alpha} \cos \left(\omega\left(\boldsymbol{Q}, \boldsymbol{Q}_{\alpha}\right)\right)\right)},
$$

where

$$
N_{\alpha}=\frac{1}{I_{0}\left(S_{\alpha}\right)-I_{1}\left(S_{\alpha}\right)}, \quad S_{\alpha}=S\left(b_{\alpha}\right)=\frac{\ln (2)}{2 \sin ^{2}\left(b_{\alpha} / 4\right)} .
$$

The parameter $b_{\alpha}$ represents the half-width of the distribution. The modified Bessel functions $I_{n}$ are defined by $I_{n}(S)=\frac{1}{\pi} \int_{0}^{\pi} \exp (S \cos (t)) \cos (n t) \mathrm{d} t$. Matthies [9] used this model function in the context of texture analysis. The following modification of the Mises-Fischer distribution takes into account the cubic crystal symmetry

$$
g\left(\boldsymbol{Q}, \boldsymbol{Q}_{\alpha}, S_{\alpha}\right)=\frac{1}{24} \sum_{\beta=1}^{24} N_{\alpha} e^{S_{\alpha} \cos \left(\omega\left(\boldsymbol{Q}, \boldsymbol{Q}_{\alpha} \boldsymbol{H}_{\beta}^{C}\right)\right)} .
$$

The $\boldsymbol{H}_{\beta}^{C} \in S O(3)$ specify the 24 elements of the symmetry group of cubic crystals. Now we define $\xi_{\alpha}$ by $\tau_{\alpha}^{C}=\xi_{\alpha} \bar{\tau}^{C}$ and take into account that the stress deviator is homogeneous of degree $1 / m$ in the rate of deformation. As a result the isotropic and the anisotropic part of the stress deviator $\overline{\boldsymbol{\tau}}^{\prime}=\overline{\boldsymbol{\tau}}^{I}+\overline{\boldsymbol{\tau}}^{\prime A}$ are given by

$$
\overline{\boldsymbol{\tau}}^{\prime I}=\int_{S O(3)} \boldsymbol{\tau}^{\prime}\left(\overline{\boldsymbol{D}}^{\prime}, \boldsymbol{Q}, \bar{\tau}^{C}\right) \mathrm{d} Q, \quad \overline{\boldsymbol{\tau}}^{\prime A}=\int_{S O(3)} \sum_{\alpha=1}^{N_{c}} \hat{\nu}_{\alpha}^{A} g\left(\boldsymbol{Q}, \boldsymbol{Q}_{\alpha}, b_{\alpha}\right) \boldsymbol{\tau}^{\prime}\left(\overline{\boldsymbol{D}}^{\prime}, \boldsymbol{Q}, \bar{\tau}^{C}\right) \mathrm{d} Q
$$

with $\hat{\nu}^{A}=\nu^{A} \nu_{\alpha}^{A} \xi_{\alpha}^{m}$. It can be seen that the weights in the orientational average of the stress tensor depend on the hardening state in the aggregate. 

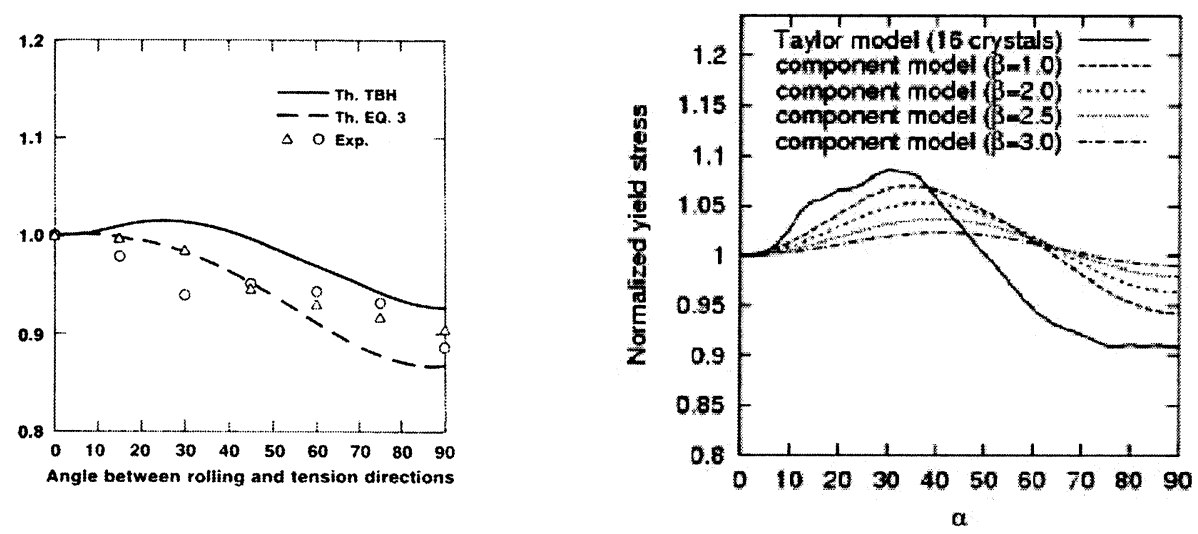

Fig. 1: Normalized yield stress versus tensile direction. Left: Numerical and experimental results by Lege et al. [8]. Right: Predictions of the texture component model and the Taylor model.

\section{Numerical Examples}

Lege et al. [8] determined the volume fractions, the half-widths, and the Euler angles of four texture components, which together with a random component, reproduce the main features of a crystallographic texture in a rolled aluminum sheet. Here we consider the problem of computing the sheet anisotropy for this set of texture components. Note that the orthotropic sample symmetry of the polycrystal requires to use 16 components. The texture component model and a Taylor type model based on discrete crystal orientations have been implemented into the commercial finite element code ABAQUS [1]. It is known that an application of the Taylor model with discrete crystal orientations would drastically overestimate the amount of anisotropy if each texture component would be modeled by only one crystal orientation. This has been shown in [2] for the yield stresses and the $R$ values. There it has also been shown that the anisotropy is also overestimated by the texture component model if the half-width is determined by the codf. This is due to the fact that the model represents an upper bound estimate of the viscoplastic behavior. These findings motivate the introduction of corrected half-width values $\tilde{b}_{\alpha}=\beta b_{\alpha}$ where $\beta \in[1,3]$. The implications of this approach are analyzed in this paper for the yield stress, the $R$ value, and the earing profile. The results are compared with the experimental data taken from [8].

In Figure 1 (right) the yield stress is shown as it is predicted by the component model and by the Taylor model. Figure 2 (right) shows the corresponding $R$ value. In the case of an application of the component model, the anisotropies are less pronounced compared to the Taylor model. If the half-width is increased, the anisotropy in terms of the yield stresses and the $R$ values decreases. In addition, if factors $\beta \approx 3$ are used, the predictions of the component model are close to the experimental results (see Figure 1 and Figure 2, left). For $\beta=1$, the results of the component model are similar to those of the Taylor-Bishop-Hill model (see Figure 1 , left).

The deep drawing of cylindrical cups is another mechanical process for which the plastic anisotropy of a sheet metal is of significant importance. For the simulations of the deep drawing test, the same components are used as in the examples discussed before. The geometry of the sheet and of the punch are taken from [8]. The orthotropic sample symmetry in the sheet plane implies that only a quarter of the sheet has to be modeled. In the calculation with ABAQUS, C3D8H elements are used. The computations have been performed for both the texture component model and the Taylor model based on discrete crystal orientations. In the 

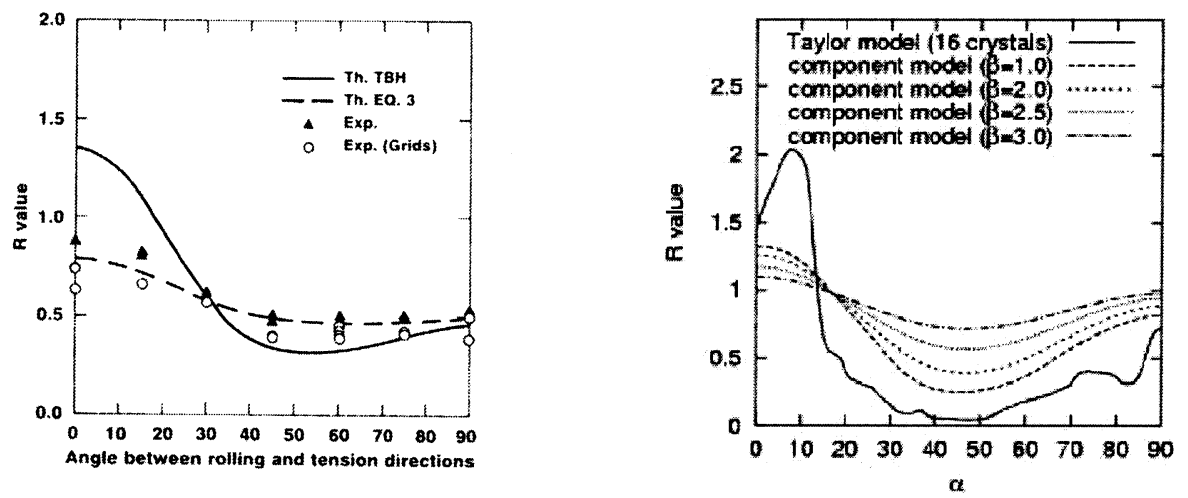

Fig. 2: $R$ values versus tensile direction. Left: Numerical and experimental results by Lege et al. [8]. Right: Prediction of the texture component model and the Taylor model.
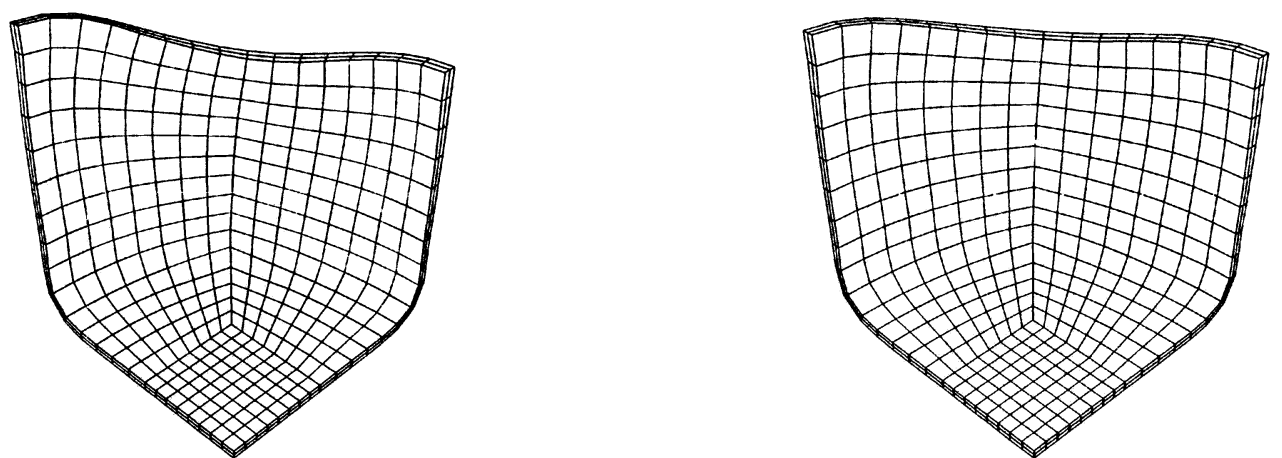

Fig. 3: Deformed finite element mesh of a quarter of a cylindrical cup. Left: Taylor model. Right: Texture component model $\left(N_{c}=4, \beta=1\right)$.

case of the component model (Figure 3, right), the maximum height of the drawn cup is smaller than in the case of the classical Taylor model (Figure 3, left). In Figure 4 (right) the influence of the parameter $\beta$ on the height of the cup is shown. If $\beta$ is increased, then the maximum height of the cup decreases.

\section{Conclusions}

A texture component based model [2] has been used to simulate the plastic anisotropy of a textured aluminum sheet by the finite element method. Special emphasis is given to the quantitatively correct modeling of the earing profile. The conclusions are the following:

- The texture component model overestimates the mechanical anisotropy of the sheet metal if the shape parameters (half-width) of the model functions are identified by an experimental codf. This is due to the fact that the component model assumes a homogeneous deformation and hence represents an upper bound estimate.

- If adjusted values of shape parameters are used then the overestimation of the anisotropy can be eliminated. It is important to note that the half-width is varied for a fixed number of model functions. This is a specific feature of an approach based on continuous model functions. If the Taylor model would be applied based on an approximation of the codf by a set of discrete crystal orientations, then the number of crystals would have to be increased in order to weaken the anisotropy. 

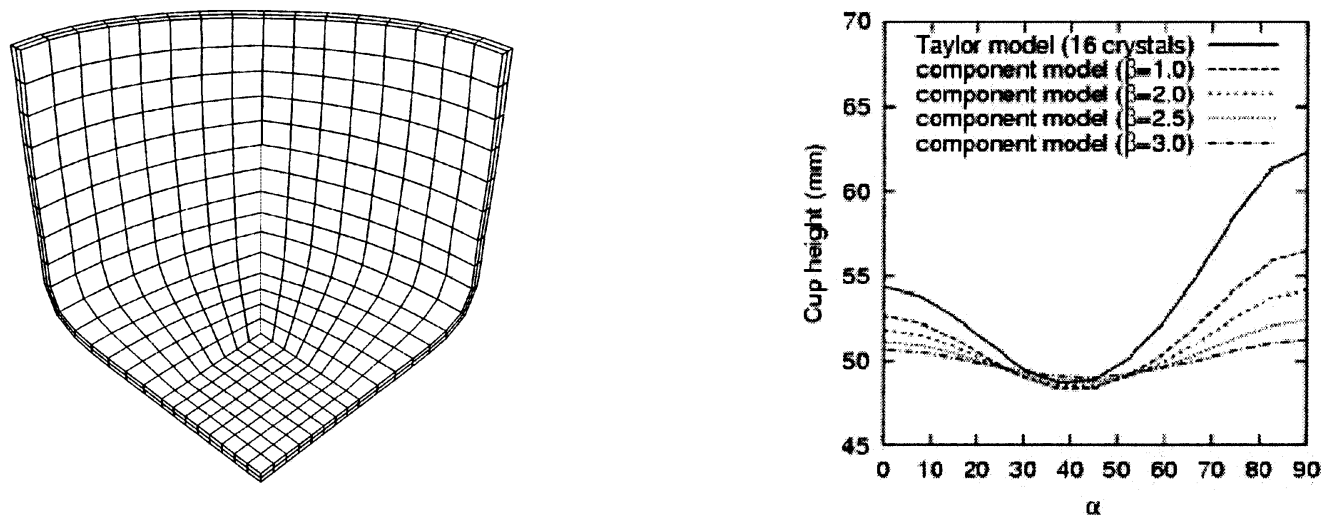

Fig. 4: Left: Deformed finite element mesh of a quarter of a cylindrical cup (texture component model with $\beta=3$ ). Right: Cup heights predicted by the texture component model and the Taylor model based on discrete crystal orientations.

Acknowledgment: Partial support for this research was provided by the Deutsche Forschungsgemeinschaft (DFG) under grant BE 1455/7 and GK 828.

\section{References}

[1] ABAQUS Standard User's Manual. Version 6.4. Hibbit, Karlsson, Sorensen, Inc., 2004.

[2] Böhlke, T., Risy, G., and Bertram, A. (2004). A texture component model for anisotropic polycrystal plasticity. To appear in Comp. Mat. Science.

[3] Bunge, H.-J. (1993). Texture Analysis in Material Science. Cuviller Verlag Göttingen.

[4] Helming, K. (1996). Texturapproximation durch Modellkomponenten. Cuvillier Verlag Göttingen.

[5] Hutchinson, J. (1976). Bounds and self-consistent estimates for creep of polycrystalline materials. Proc. R. Soc. Lon., A 348:101-127.

[6] Kocks, U. and Mecking, H. (2003). Physics and phenomenology of strain hardening: The FCC case. Progr. Mat. Sci., 48:171-273.

[7] Kocks, U., Tome, C., and Wenk, H. (1998). Texture and Anisotropy: Preferred Orientations in Polycrystals and Their Effect on Materials Properties. Cambridge Univ. Pr.

[8] Lege, D., Barlat, F., and Brem, J. (1989). Characterization and modelling of the mechanical behavior and formability of a 2008-T4 sheet sample. Int. J. Mech. Sci., 31(7):549-563.

[9] Matthies, S., 1980. Standard functions in texture analysis. Phys. Stat. Sol. B 101, K111-K115.

[10] Simo, J., Miehe, C., 1992. Associative coupled thermoplasticity at finite stains: Formulation, numerical analyses and implementation. Comp. Meth. Appl. Mech. Engng. 98, 41-104.

[11] Wasserman, G. and Grewen, J. (1962). Texturen metallischer Werkstoffe. Springer. 
Textures of Materials - ICOTOM 14

doi:10.4028/www.scientific.net/MSF.495-497

A Texture Based Model for Polycrystal Plasticity doi:10.4028/www.scientific.net/MSF.495-497.1091 